\title{
Jet Noise Receptivity to Nozzle-upstream Perturbations in Compressible Heated Jets
}

\author{
Yee Chee See, Ghobad Amini† Christopher Koh and Matthias Ihme ${ }^{\S}$ \\ Department of Aerospace Engineering \\ University of Michigan \\ Ann Arbor, MI 48109
}

\begin{abstract}
Effects of nozzle-upstream entropy perturbations on the acoustic radiation from heated jets are investigated. For this, a model problem is considered, in which a gas-turbine combustor discharges reaction products through a converging nozzle into the ambient environment. The turbulent reacting flow field in the combustor is computed using large-eddy simulation (LES), and the unsteady flow-field at the combustor exit is extracted to provide realistic inflow conditions to the jet-flow simulation. To study the indirect coupling process, arising from the interaction of the combustion-generated entropy fluctuations with the adverse pressure gradient through the nozzle, a linearized Euler formulation is employed. Parametric studies are performed to investigate effects of frequency and amplitude of the nozzle-upstream entropy perturbations on the jet instability and the jet noise directivity. Simulation results show that the directivity is dependent on the perturbation frequency. Excitation near the preferred shear-layer instability leads to strong acoustic radiation in the $45^{\circ}$ forward direction, and the radiation angle decreases with decreasing excitation frequency.
\end{abstract}

\section{Introduction}

Jet exhaust noise is a major contributor to the far-field radiation in low-bypass aircraft engines. Over the last thirty years, significant progress has been made in understanding the noise-generating mechanisms of subsonic and supersonic jets. ${ }^{1-3}$ Specifically, the noise from imperfectly expanded jets can be separated in contributions from turbulent mixing noise, broadband shock-associated noise, and screech tones. The relative intensity of these individual noise source components is dependent on operating conditions.

Apart from the direct propagation of core noise to the far field, core noise fluctuations can play a pivotal role in the jet noise amplification. ${ }^{4}$ Experimental studies have shown that tonal and broadband excitations upstream of the nozzle exit can amplify the jet noise by as much as 5-10 $\mathrm{dB}$ compared to the unforced condition. ${ }^{5-7}$ In particular, Bechert \& Pfizenmaier ${ }^{7,8}$ reported a pronounced broadband shift of the radiated sound for excitations at the preferred shear-layer frequency. These observations on the jet noise amplification were confirmed by Berman ${ }^{9}$ for axial and coaxial low-bypass jets; they attributed this so-called excess noise to the excitation of the shear layer and subsequent increase in turbulent stresses. Comprehensive experimental investigations by $\mathrm{Lu}^{5}$ in coaxial jets at simulated high bypass ratio engine conditions showed that also broadband excitations can generate significant excess jet noise, which increases with increasing excitation level.

Although detailed understanding and comprehensive data about excess noise in heated and imperfectly expanded jets are largely missing, an experimental study by Jubelin ${ }^{10}$ concluded that heated jets are more receptive to upstream excitations, and the generated excess noise has a pronounced directivity in the forward direction. Furthermore, measurements in supercritical jets showed that shock-associated noise can be

\footnotetext{
* Research Assistant, Department of Aerospace Engineering, University of Michigan, AIAA member

${ }^{\dagger}$ Post-doctoral Scholar, Department of Aerospace Engineering, University of Michigan, AIAA member

${ }^{\ddagger}$ Research Assistant, Department of Aerospace Engineering, University of Michigan, AIAA member

$\S$ Assistant Professor, Department of Aerospace Engineering, University of Michigan,AIAA member
} 
strongly amplified. Compared to subsonic jets, these far-field spectra did not show evidence of the tonal excitation, which suggests that the absence of irregularities in the spectral shape is insufficient to discriminate between excited and unexcited jet noise. ${ }^{10}$

Toward investigating the effect of turbulence on the jet-acoustic response, experimental investigations by Raman et al. ${ }^{11}$ revealed that the frequency of the preferred mode and excitation amplitude depends on the initial level of turbulence intensity. Comprehensive investigations by Ahuja and coworkers examined the jet noise broadband amplification problem using flow visualization, ${ }^{12}$ flow measurements ${ }^{12,13}$ and acoustic measurements. ${ }^{14}$ They concluded that amplified large-scale structures result in increased small-scale turbulence and the small-scale turbulence is responsible for the increased broadband noise. ${ }^{14}$ Theoretical results by Zaman showed that excitations enhance the vortex pairing, which is responsible for the increased sound generation. ${ }^{15}$

The objective of this work is to investigate effects of nozzle-upstream perturbations on the acoustic radiation in heated jets. To this end, large-eddy simulations of a model gas-turbine combustor are performed to provide realistic combustor exit conditions. The combustor exit is connected to a converging nozzle, discharging the combustion products into the ambient environment. Details regarding the model problem and computational configuration are given in the next section. A linear Euler formulation is used to model the indirect noise-generation that results from the coupling between entropy inhomogeneities exiting the combustor and pressure radiation. The computational model for the description of the combustor, the jet mean-flow simulation, and linearized Euler formulation are discussed in Sec. III. Modeling results are presented in Sec. IV, and the paper finishes with conclusions.

\section{Problem Formulation}

In aircraft engines several acoustic interaction processes exists, and the underlying coupling mechanisms are schematically illustrated in Fig. 1. Specifically, the unsteady flow-field in the combustor generates inhomogeneities in vorticity, pressure, and entropy. Pressure perturbations that are generated in the engine are commonly associated with direct-noise contributions that propagate downstream, exit the nozzle, and radiate to the far-field. Although dependent on engine configuration and operating regime, these direct core noise contributions typically fall in the low-frequency range, corresponding to

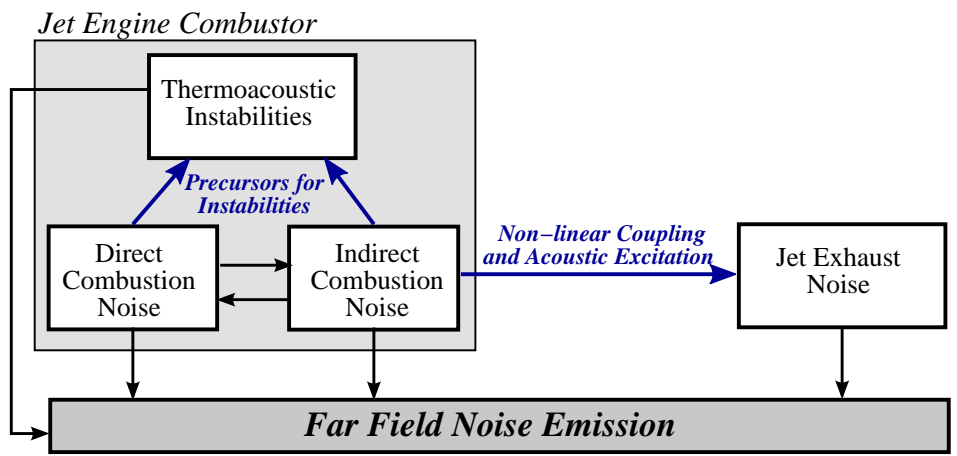

Figure 1. Direct and indirect noise source contributions originating from unsteady combustion process, non-linear coupling mechanisms, and jet noise amplification; Arrows in blue show the indirect core noise contributions.

the $200-400 \mathrm{~Hz}$ frequency band. ${ }^{16,17}$ In this context it is also noted that these direct noise-sources can act as a bypass mechanism to excite thermo-acoustic instabilities in the combustor, ${ }^{18-21}$ and even relatively weak interactions can trigger combustion instabilities. ${ }^{22}$

Apart from combustion-generated pressure perturbations, the unsteady combustion process generates velocity and entropy fluctuations that exit the combustor, and are convected to the nozzle. As shown by Marble \& Candel ${ }^{23}$ these entropy perturbations interact with the pressure gradients in the turbine and the nozzle, and are converted to acoustic pressure perturbations. This process is referred to as indirect noise.

The present work is concerned with investigating effects of entropy perturbations on the acoustic radiation in heated jets. A schematic of the model-problem is illustrated in Fig. 2, and consists of a gas-turbine combustor which is connected to a converging nozzle, expanding the combustor exhaust products into the ambient environment. Large-eddy simulations of the combustor are performed to evaluate the mean-flow and fluctuating quantities at the combustor exit. The mean-flow in the nozzle and the jet is obtained as solution of a steady-state Reynolds-averaged Navier-Stokes (RANS) simulation, and the spatio-temporal evolution of the perturbations of pressure, velocity, and entropy are evaluated from the linearized Euler equations (LEE). Details on the mathematical model, geometric configurations, and operating conditions are provided in the next section. 


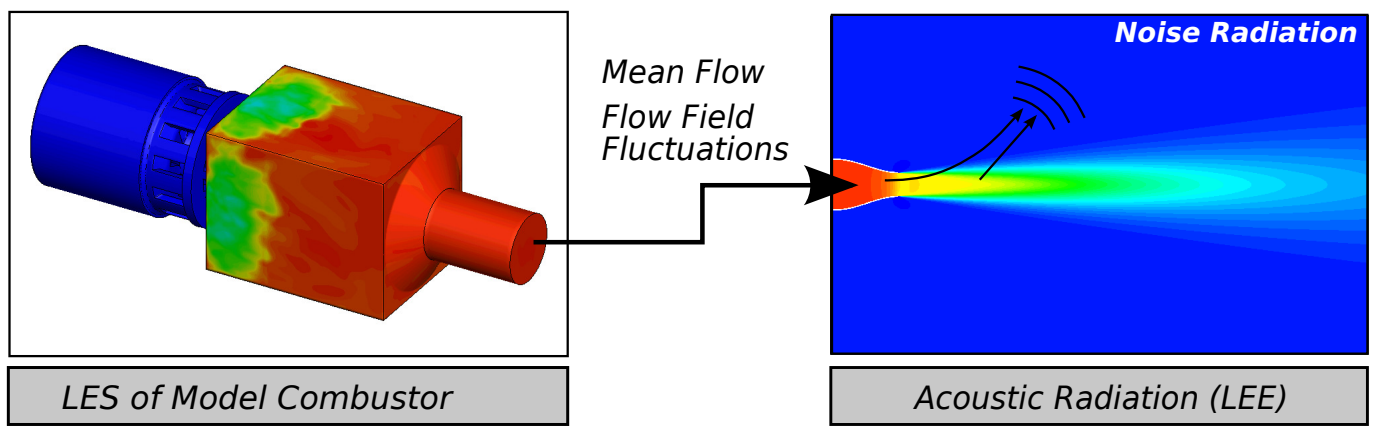

Figure 2. Schematic of the model-problem, consisting of (i) a large-eddy simulation of a model gas turbine combustor for predicting transient combustor exit conditions, and (ii) simulation of the nozzle flow and acoustic far-field radiation.

\section{Computational Model}

\section{A. Gas Turbine Combustor Model}

In this work, we consider the gas turbine model combustor (GTMC) that was experimentally investigated by Meier et al. ${ }^{24,24}$ A schematic of the burner is illustrated in Fig. 3. The injector consists of a central air nozzle, an annular fuel nozzle, and a co-annular air nozzle. Both air nozzles supply swirling air at ambient temperature from a common plenum. The inner air nozzle has a diameter of $15 \mathrm{~mm}$; the annular nozzle has an inner diameter of $17 \mathrm{~mm}$ and an outer diameter of 25 $\mathrm{mm}$. The measured swirl number is 0.55 . Non-swirling fuel is provided through three exterior ports fed through the annular nozzle. The exit plane of the central air nozzle and fuel nozzle lies $4.5 \mathrm{~mm}$ below the exit plane of the outer air annulus. The combustion chamber has a square cross section of $85 \mathrm{~mm}$ in width and $110 \mathrm{~mm}$ in height. The exit of the combustion chamber is an exhaust tube with a diameter of $40 \mathrm{~mm}$ and a height of $50 \mathrm{~mm}$.

The spatio-temporal evolution of the unsteady turbulent flow-field is obtained from the solution of a low-Mach number variable density formulation. Turbulent combustion, heat-transfer, and species conversion are described using a flamelet/progress variable (FPV) formulation. ${ }^{25,26}$ In this combustion model, all thermochemical quantities are parameterized by a two-scalar manifold, consisting of mixture fraction $Z$ (which accounts for the mixing of reactants) and a progress variable $C$ to represent the progress of reaction and fuel conversion.

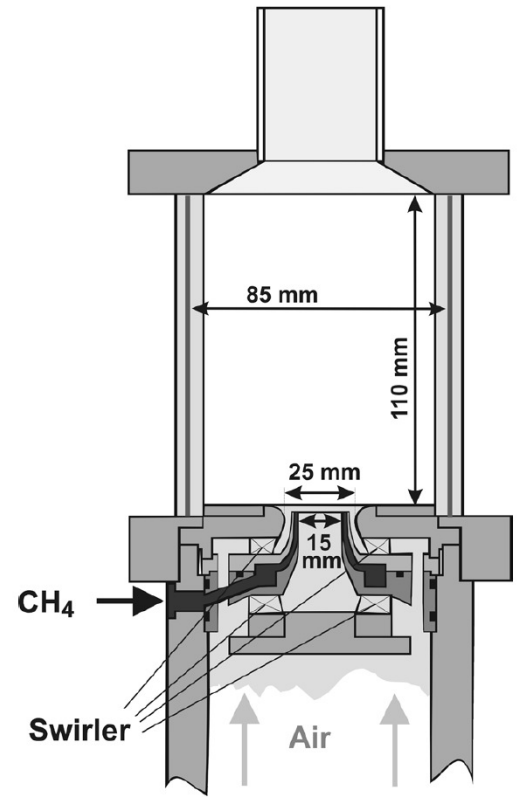

Figure 3. Schematic of gas turbine model combustor. ${ }^{24,24}$ Turbulence/chemistry interaction, occurring on the computationally unresolved scales, is modeled using a presumed PDF-closure.

The entire burner, consisting of plenum, swirler, combustion chamber, and exhaust, is discretized using a hexahedral mesh. The regular geometry of the plenum, combustion-chamber, and exhaust allows for utilizing a regular block-structured grid, and an unstructured grid is employed for discretizing the swirler-module. The computational mesh consists of 5.5 million control volumes. Although not further considered in this contribution, a grid-convergence study was performed to assess the sensitivity of the numerical solution to the mesh-resolution.

In the present simulation the stably-operating burner configuration, designed as "flame A", ${ }^{24}$ is considered. In this configuration, the flame is stabilized and no acoustic coupling was experimentally observed. The burner is operated with methane and its thermal power output is $34.9 \mathrm{~kW}$. The air mass-flow rate is 1095 $\mathrm{g} / \mathrm{min}$ and fuel is supplied at a mass flow-rate of $41.8 \mathrm{~g} / \mathrm{min}$, corresponding to an overall equivalence ratio of $\Phi=0.65$. The reaction chemistry is described by a detailed methane reaction mechanism, ${ }^{27}$ consisting of 279 reactions among 49 species. 
Computational results, obtained from this large-eddy simulation, are presented in Fig. 4. The instantaneous axial velocity field is shown in Fig. 4(a). Air is entering the combustor at the bottom through the plenum. By passing through the swirlers, tangential momentum is imparted on the fluid, which induces shear and promotes rapid mixing with the fuel stream after exiting the swirl-nozzle. The rapid mixing between reactants can also be observed from the instantaneous mixture fraction field that is shown in Fig. 4(b). The black solid line corresponds to the isocontour of stoichiometric mixture, and can be associated with the location of maximum heat release.

The instantaneous temperature field is shown in Fig. 4(c). From this figure it can be deduced that the flame is almost planar and anchored near the nozzle. Recirculation zones in the corner region and upstream of the nozzle are evident. By entraining cold fluid and reducing the strain-rate, these regions promote mixing of air with hot reaction products and stabilize the flame. With increasing downstream distance, reactants and products mix, leading to a nearly homogeneous mixture at the combustor exit.

The combustion products are discharged through a converging nozzle into the ambient environment, and effects of transient combustor exit conditions on the acoustic radiation are investigated. The computation of the nozzle-flow and simulation of the acoustic radiation using a linearized Euler formulation are presented in the next section.

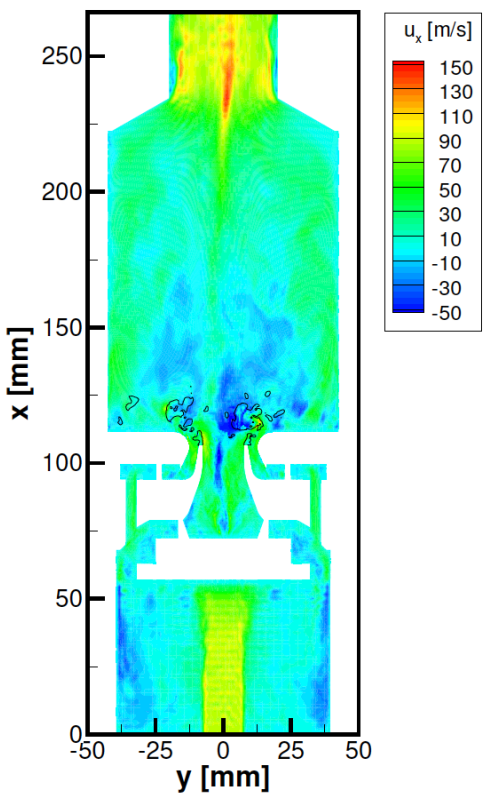

(a) Normalized axial velocity

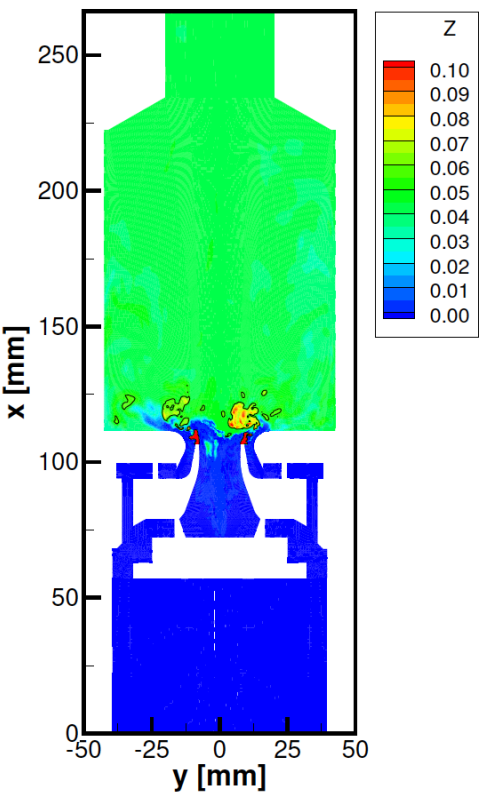

(b) Mixture fraction

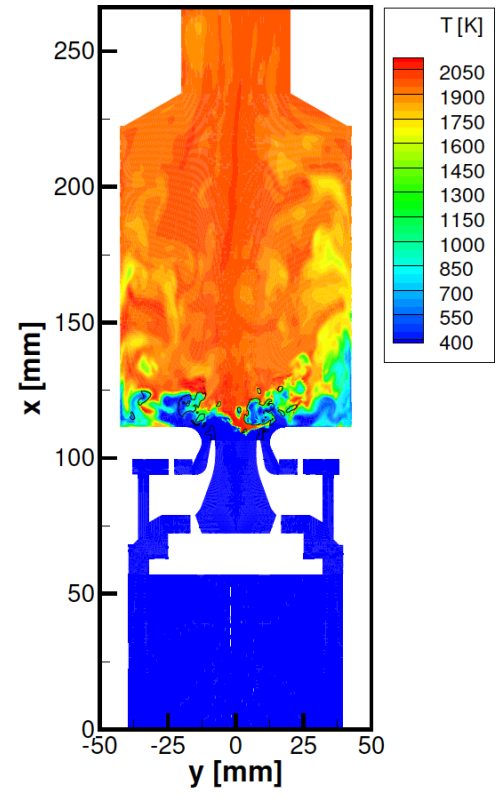

(c) Temperature

Figure 4. Instantaneous flow-field results along a cross-sectional plane through the gas turbine model combustor, showing (a) axial velocity (b) mixture fraction, and (c) temperature. The solid line corresponds to the isocontour of stoichiometric mixture.

\section{B. Jet Mean Flow Computation}

The exit of the combustor is connected to a converging nozzle, and a separate steady-state RANS computation is performed to compute the flow in the nozzle and the heated jet. This RANS simulation begins at the nozzle inlet, at which flow-field conditions from the combustor simulation are prescribed. The nozzle inlet conditions for the mean-flow simulation are obtained by averaging over the combustor exit area, resulting in a mean temperature of $\langle\widetilde{T}\rangle=1752 \mathrm{~K}$ and mean pressure of $\langle\bar{p}\rangle=2$ bar (corresponding to a combustor exit Mach number of 0.123).

The geometry of the nozzle is approximated by a cubic polynomial, having the following form

$$
r=172.8 x^{3}+12.96 x^{2}+0.0092[\mathrm{~m}] \quad \text { for } \quad-0.05 \mathrm{~m} \leq x \leq 0,
$$

where $r$ and $x$ are the radial and axial coordinates. The nozzle has zero slopes at both ends and a thickness of $1 \mathrm{~mm}$ with a round trailing edge. The nozzle geometry together with the geometry-conformal mesh around 
the nozzle are illustrated in Fig. 5. Based on the combustor exit conditions and the nozzle geometry, the

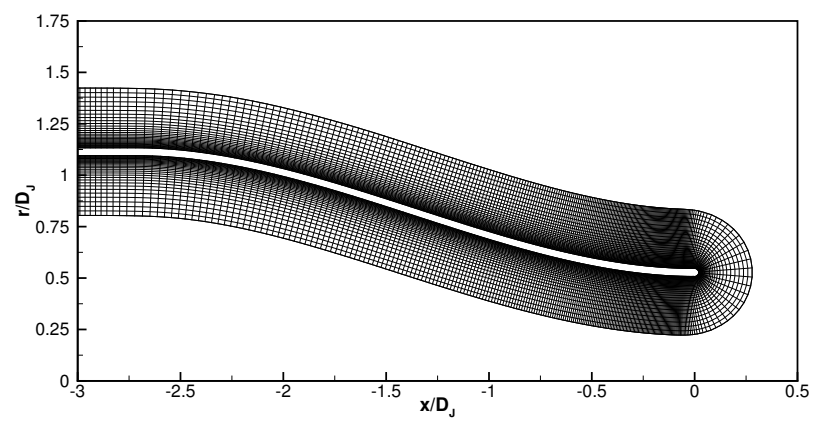

Figure 5. Nozzle geometry and geometry-conformal mesh around the nozzle geometry.

nozzle exit Mach number is 0.95 and the Reynolds number, $\operatorname{Re}=U_{J} D_{J} / \nu$, is 200,000. The computational domain for the jet mean-flow simulation extends up to $x=1 \mathrm{~m}$ and the spatial extend in radial direction is $r=0.5 \mathrm{~m}$, corresponding to 54 and 27 nozzle exit diameters, respectively. The entire computational domain was discretized using 300,000 control volumes, and a grid-refinement study was performed to ensure that the solution is grid-independent. A geometry-conformal structured mesh is used within the nozzle and an unstructured grid is used to discretize the nozzle-exterior domain. Local grid refinement is employed in the shear-layer region. A finite volume method is used to solve the compressible Navier-Stokes equations in an axisymmetric domain. The steady RANS equations are solved using a second-order upwind flow solver with standard $k-\varepsilon$ turbulence model. Pressure boundary conditions are enforced at the outlet, and gauge pressure of 1 bar and temperature of $1752 \mathrm{~K}$ are prescribes as inlet conditions.

Figure 6 compares simulation results from the 2D RANS computation with analytical results that are obtained from 1D nozzle theory. Overall, good agreement is obtained, and small differences near the nozzle exit are attributed to the formation of expansion fans as results of the under-expanded nozzle exit condition. Shown in the middle figure is the temperature along the nozzle axis. Temperature is normalized by the ambient condition. Corresponding to the combustor operating conditions and the nozzle geometry, the temperature ratio at the nozzle exit is $T_{J} / T_{0}=5$.
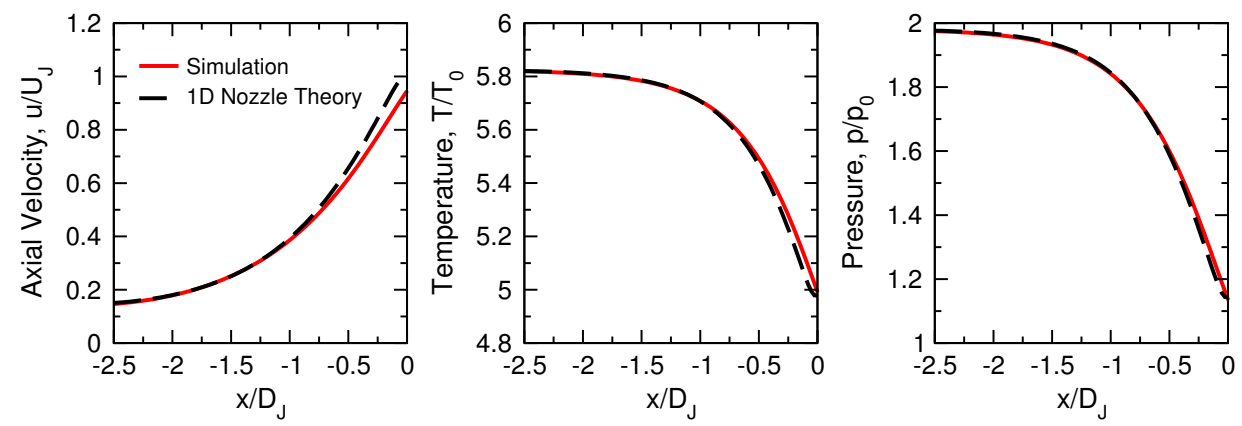

Figure 6. Comparison of flow-predictions through the nozzle: axial velocity (left), temperature (middle), and pressure (right).

Due to the under-expanded nozzle condition (see right of Fig. 6), an expansion fan forms at the nozzle lip, resulting in velocity oscillations near the nozzle. This is illustrated in Fig. 7, showing the mean axial velocity along the jet centerline. To facilitate a qualitative comparison of the simulation results, we also present measurements that were reported by Plumblee et al. ${ }^{28}$ It is noted that exit condition and heating ratio are not representative for the configuration under investigation, but nevertheless enables a relative comparison.

The mean axial velocity field and mean temperature field are shown in Fig. 8. Velocity contours through the nozzle indicate the presence of two-dimensional effects, and the presence of a shock-cell near the nozzle exit is observable. The mean temperature field is shown on the right of Fig. 8. Due to the expansion through the nozzle, the temperature reduces by approximately $250 \mathrm{~K}$. The temperature affects the thermoviscous 


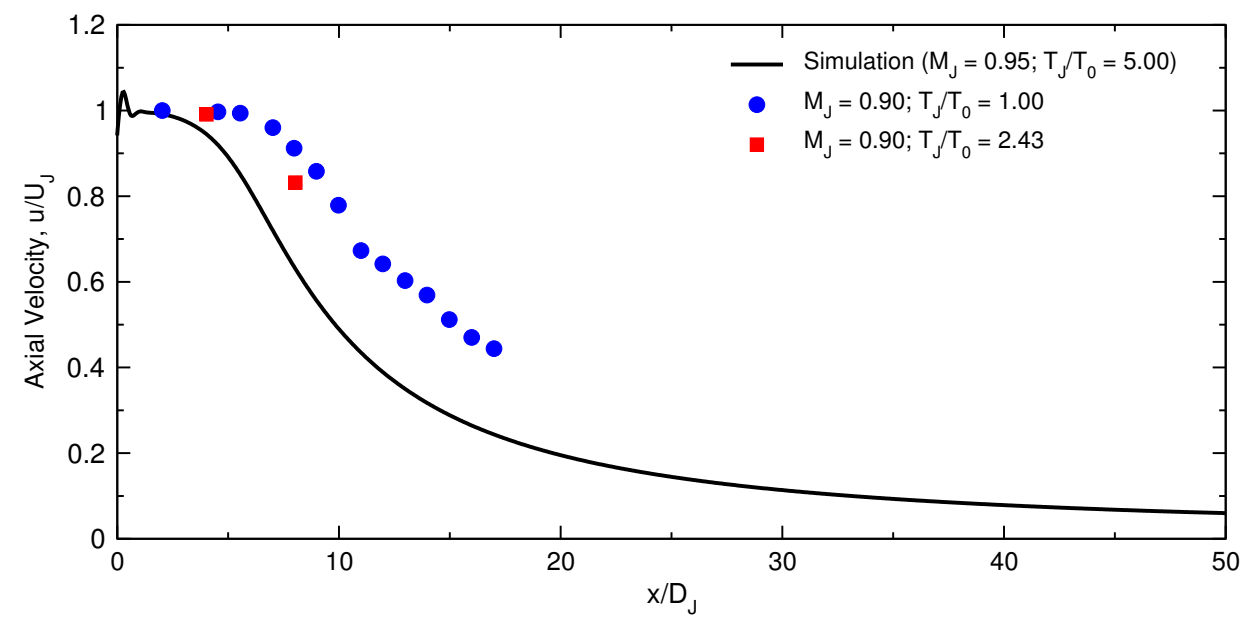

Figure 7. Axial velocity along jet centerline. Experimental data ${ }^{28}$ for qualitative comparison are shown by symbols.

properties, increasing the viscosity and thereby suppressing the turbulence generation. Theoretical analysis by Monkewitz \& Sohn ${ }^{29}$ suggests that heated jets can exhibit regions of absolute instability in the potential core, and this stability boundary is dependent on operating conditions and density ratio. Regions of absolute instability can lead to exponential growth of perturbations that are generated upstream of the nozzle exit. These absolute instability regions can enhance the noise generation, and this issue will be addressed in the next section.
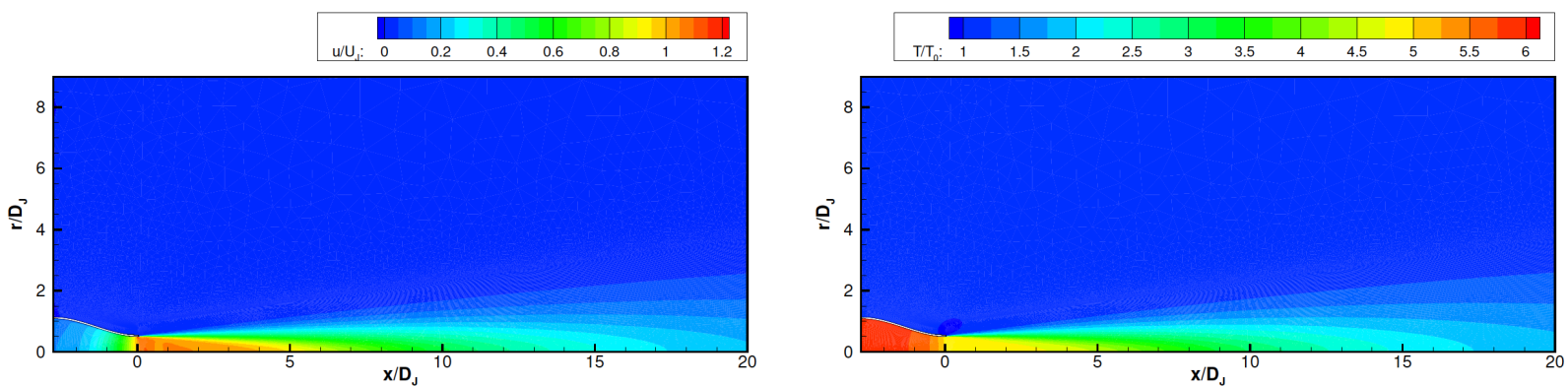

Figure 8. Predicted jet mean flow field: axial velocity (left) and temperature (right).

\section{Linearized Euler Model}

\section{Mathematical Model}

The evolution of the jet flow is governed by the unsteady Navier-Stokes equations which are here written for pressure $p$, velocity $\boldsymbol{u}$, and entropy $s$. These equations can be written in non-dimensional form as:

$$
\begin{aligned}
\partial_{t} p+\boldsymbol{u} \cdot \nabla p+\gamma p \nabla \cdot \boldsymbol{u} & =(\gamma-1)[\mathcal{C}+\mathcal{D}], \\
\partial_{t} \boldsymbol{u}+\boldsymbol{u} \cdot \nabla \boldsymbol{u} & =-\frac{1}{\gamma \mathrm{M}^{2}} \frac{1}{\rho} \nabla p+\frac{1}{\operatorname{Re}} \frac{1}{\rho} \nabla \cdot \underline{\underline{\tau}}, \\
\partial_{t} s+\boldsymbol{u} \cdot \nabla s & =\frac{1}{p}[\mathcal{C}+\mathcal{D}],
\end{aligned}
$$

where $\mathcal{C}=\frac{\gamma}{\gamma-1} \frac{1}{\operatorname{Re} \operatorname{Pr}} \nabla \cdot(\lambda \nabla T)$ is the heat flux, $\mathcal{D}=\gamma \frac{\mathrm{M}^{2}}{\operatorname{Re}} \underline{\underline{\tau}}: \nabla \boldsymbol{u}$ is the viscous dissipation, and $\underline{\underline{\tau}}$ is the viscous stress tensor.

To obtain the linearized Euler equations (LEE), the state vector $\boldsymbol{\phi}(\boldsymbol{x}, t)=(p, \boldsymbol{u}, s)^{T}$ is decomposed into mean and fluctuating components, $\phi(\boldsymbol{x}, t)=\bar{\phi}(\boldsymbol{x})+\boldsymbol{\phi}^{\prime}(\boldsymbol{x}, t)$, in which an over-bar denotes a mean quantity 
and the prime refers to a perturbation. After expanding Eqs. (2), and neglecting viscous and higher-order terms, the linearized Euler equations can be written as:

$$
\begin{aligned}
\partial_{t} p^{\prime}+\boldsymbol{u}^{\prime} \cdot \nabla \bar{p}+\overline{\boldsymbol{u}} \cdot \nabla p^{\prime}+\gamma\left(p^{\prime} \nabla \cdot \overline{\boldsymbol{u}}+\bar{p} \nabla \cdot \boldsymbol{u}^{\prime}\right) & =0, \\
\partial_{t} \boldsymbol{u}^{\prime}+\frac{\rho^{\prime}}{\bar{\rho}} \overline{\boldsymbol{u}} \cdot \nabla \overline{\boldsymbol{u}}+\boldsymbol{u}^{\prime} \cdot \nabla \overline{\boldsymbol{u}}+\overline{\boldsymbol{u}} \cdot \nabla \boldsymbol{u}^{\prime} & =-\frac{1}{\gamma \mathrm{M}^{2}} \frac{1}{\bar{\rho}} \nabla p^{\prime}, \\
\partial_{t} s^{\prime}+\boldsymbol{u}^{\prime} \cdot \nabla \bar{s}+\overline{\boldsymbol{u}} \cdot \nabla s^{\prime} & =0 .
\end{aligned}
$$

The density fluctuation $\rho^{\prime}$, appearing in Eq. (3), is not an independent state variable, and is related to pressure and entropy through the following expression:

$$
\frac{\rho^{\prime}}{\bar{\rho}}=\frac{1}{\gamma} \frac{p^{\prime}}{\bar{p}}-\frac{\gamma-1}{\gamma} s^{\prime} .
$$

Similarly, the temperature perturbations can be related to pressure and entropy as:

$$
\begin{aligned}
\frac{T^{\prime}}{\bar{T}} & =\frac{p^{\prime}}{\bar{p}}-\frac{\rho^{\prime}}{\bar{\rho}}, \\
& =\frac{\gamma-1}{\gamma}\left(\frac{p^{\prime}}{\bar{p}}+s^{\prime}\right) .
\end{aligned}
$$

\section{Numerical Method}

The linearized Euler equations are solved numerically in a generalized axisymmetric coordinate system. All spatial derivatives are discretized using a 4th-order dispersion-relation-preserving (DRP) scheme, ${ }^{30}$ and a low dissipation dispersion Runge-Kutta (LDDRK) scheme is used for time-advancement. ${ }^{31}$ The DRP scheme, as implemented in the solver, uses a central difference stencil in the interior of the computational domain. However, a one-sided biased stencil is used near the boundaries. The biased stencil allows for the treatment of wall boundary conditions, as proposed by Tam \& Dong. ${ }^{32}$ In this method, impermeable boundary conditions at solid walls are enforced through the pressure in the ghost-cell region. Symmetry boundary conditions are applied along the centerline. Boundary conditions at the inlet and the far-field are described using the characteristic boundary conditions. In addition to these nonreflecting boundary conditions, a sponge zone is included to further dampen any spurious reflections. Although this boundary treatment may be less effective than more sophisticated methods such as a perfectly matched layer (PML), it is applicable to more general configurations and was successfully employed by Barone \& Lele. ${ }^{33}$ Time-dependent perturbations at the inlet were imposed through the sponge layer and the sponge strength was adjusted to minimize phase-lag and retain the correct perturbation amplitude.

Due to the geometric complexity of the nozzle it is difficult to utilize a single curvilinear mesh without introducing grid singularities. To address this issue, an overset mesh-strategy is utilized, and two overlapping curvilinear grids are used. The region surrounding the nozzle is discretized using a C-grid, and a stretched Cartesian grid is used to discretize the rest of the computational domain. The exchange of flow-field information between both grids is accomplished through a 6th-order accurate interpolation scheme using the OverTure-framework, ${ }^{34}$ and a 6 th-order implicit filter ${ }^{35}$ is used to suppress spurious oscillations.

\section{LEE Verification}

The LEE-solver that was outlined in the previous section is verified using a benchmark configuration that describes the acoustic radiation of a harmonic point source which is immersed in a parallel shear-flow. ${ }^{36}$ Parameters and operating conditions are identical to the benchmark case, and results of this verification study are presented in Fig. 9. Figure 9(a) shows the instantaneous pressure field; evident from this figure is the presence of a convective Kelvin-Helmholtz instability that propagates in the jet forward direction, and an acoustic mode that radiates in the sideline direction. A comparison of simulation results and analytical results along the sideline $y=15 \mathrm{~m}$ at the beginning of the excitation period is shown in Fig. 9(b). Overall, the numerical results are in good agreement with the analytical results except for the region $x>70 \mathrm{~m}$. This discrepancy can be attributed to the formation of a shear-layer instability (see Fig. 9(a)), which is not contained in the analytical solution. 


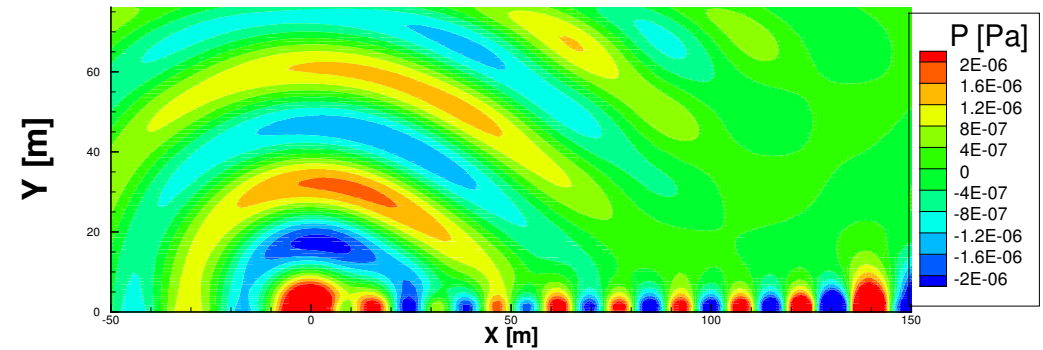

(a)

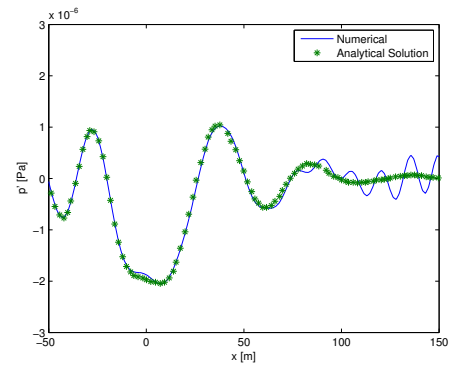

(b)

Figure 9. Verification of LEE-solver: (a) Pressure field at the beginning of an oscillation period, (b) Quantitative comparison between numerical results and analytical solution. ${ }^{36}$

\section{Results}

\section{A. Computational Configuration}

The computational domain for the LEE simulation is schematically illustrated in Fig. 10. The axial length of the simulation domain, $L_{x}$, is $0.5 \mathrm{~m}$ and its radial extend is $L_{r}=0.2 \mathrm{~m}$. The background Cartesian mesh uses 504 grid points in the stream-wise direction and 168 grid points in the radial direction. The C-grid around the nozzle has 480 grid points along the nozzle geometry and 30 grid points normal to the nozzle-wall. The shaded region in Fig. 10 indicates the sponge-layer, which is extruded into the domain. The dimension of the sponge zone and the corresponding sponge strength parameter $\sigma$ are summarized in Tab. 1. Following a quadratic decay-law, the forcing strength in the sponge zone decrease from the prescribed value of $\sigma$ at the outer end of the sponge layer to zero at the interface with the interior domain.

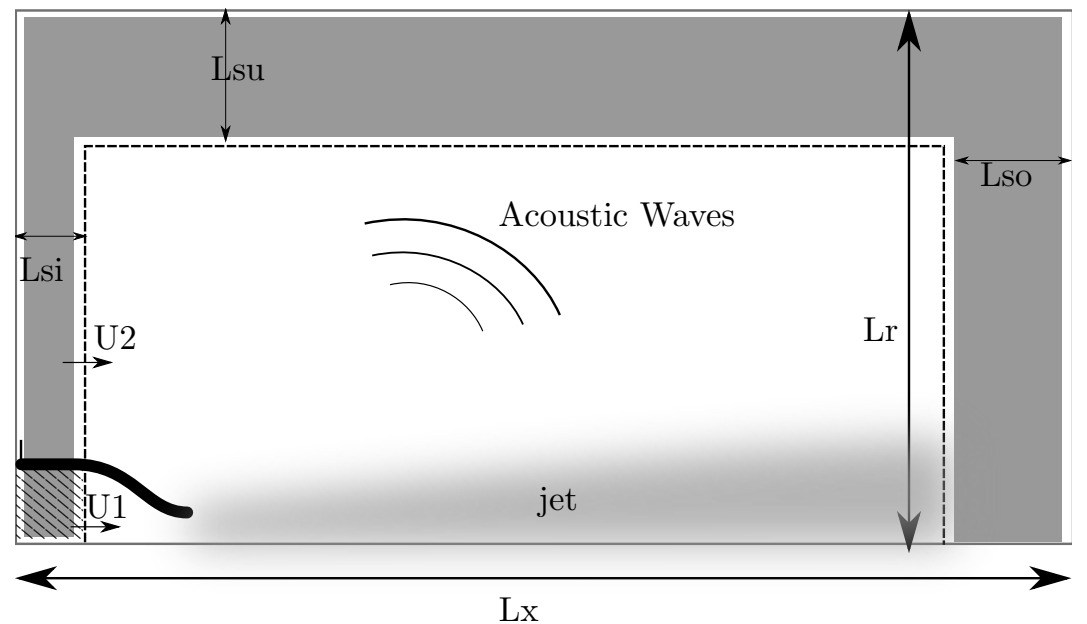

Figure 10. Schematic of the computational domain.

\begin{tabular}{|c|c|c|c|c|c|}
\hline$L_{s i}$ & $L_{s o}$ & $L_{s u}$ & $\sigma_{s i}$ & $\sigma_{s o}$ & $\sigma_{s u}$ \\
\hline \hline $0.015 \mathrm{~m}$ & $0.05 \mathrm{~m}$ & $0.1 \mathrm{~m}$ & $10^{5}$ & 10 & 10 \\
\hline
\end{tabular}

Table 1. Sponge zone parameters.

\section{B. Specification of Boundary and Mean-flow Conditions}

Transient inflow conditions at the nozzle inlet are obtained from the solution of the combustion-simulation, which was discussed in Sec. A. Since this simulation employs a low-Mach number formulation, information 
about pressure perturbations are not available, and only entropy inhomogeneities are prescribed as nozzleupstream flow-field perturbations in the LEE-model. To this end, the time history of the density field at the combustor exit was recorded, and a proper orthogonal decomposition was used to extract the first energetic radial mode. From this model the corresponding entropy perturbation profile is evaluated using the linearized thermodynamic relation of Eq. (4). Harmonic oscillations of this entropy mode of the form

$$
s^{\prime}(r, t)=\widehat{s}(r) \cos (\mathrm{St} 2 \pi t)
$$

are then enforced at the inlet. In this equation, St is the Strouhal number, $t$ is the non-dimensional time, and the first energetic entropy POD-mode $\widehat{s}(r)$ is shown in Fig. 11. This time-dependent boundary condition is prescribed at the nozzle inflow. In the following, three different forcing frequencies, corresponding to St $=\{0.255,0.051,0.0255\}$, are considered, and simulation results are presented in the next section.

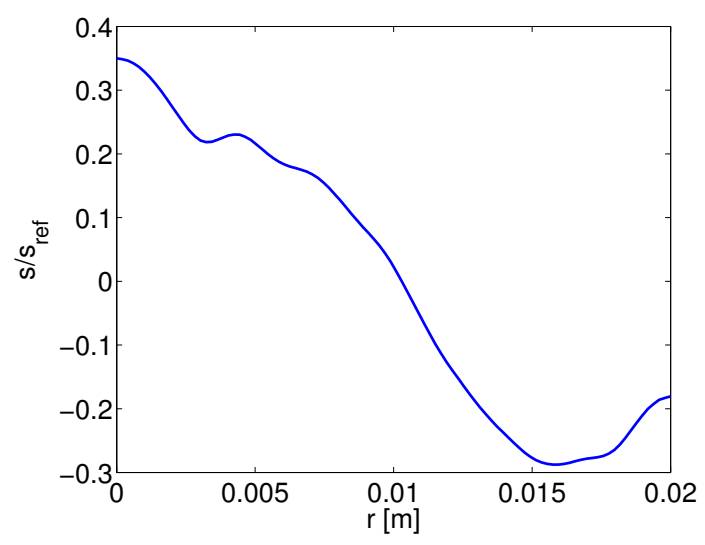

Figure 11. Entropy mode $\widehat{s}(r)$ obtained from POD-analysis.

\section{Results and Discussion}

In this section, modeling results from the LEE-formulation are presented. The reader is reminded that this investigation focuses on the flow-field response to nozzle-upstream perturbations, and non-linear noise-source mechanisms, arising from the turbulence/acoustic/mean-flow interaction are not considered. A numerical investigation of the impulse response showed that the jet-flow is convectively unstable, and an absolutely unstable behavior was not observed for this particular operating condition.

Instantaneous flow-field results for pressure and entropy at three different Strouhal numbers are presented in Fig. 12. Entropy perturbations at the nozzle inlet interact with the pressure gradient through the nozzle. This coupling process leads to the generation of pressure and velocity instabilities that propagate as acoustic and convective waves, respectively. The spatial evolution of the pressure and entropy waves in the jet-near field shows that the directivity and growth-rate of the instability waves are dependent on the excitation frequency. The configuration with the highest excitation frequency of $\mathrm{St}=0.255$, shown in the first row of Fig. 12, exhibits a pronounced directivity of the pressure radiation in the $45^{\circ}$ forward direction. Unlike the pressure, the entropy-field, shown in Fig. 12(b), only exhibits a weak growth, and the perturbations decay beyond $x / D_{J}=15$. The excitation frequency of $\mathrm{St}=0.255$ is close to the preferred shear-layer instability. A reduction of the excitation frequency by a factor of 5 and 10, respectively, leads to a shift in the directivity towards the jet forward direction and enhanced amplification of the hydrodynamic instabilities in jet-downstream direction. Figures $12(\mathrm{~d})$ and $12(\mathrm{f})$ show that the entropy waves are stretched along the axial direction and are amplified for $x / D_{J}>10$.

A comparison of the pressure directivity in the jet near-field confirms the frequency-dependent jet noiseradiation. This comparison is illustrated in Fig. 13, showing the root-mean-square pressure signal at a radial distance of $r / D_{J}=8.5$ along the axial direction. This comparison confirms the frequency-dependent shift in the directivity, and the pressure radiation shifts towards the jet-forward direct with lower frequency. This requires further consideration and is addressed in subsequent work.

A comparison of the pressure signal and power spectral density at a measurement location of $x / D_{J}=15$ and $r / D_{J}=8$ is presented in Fig. 14. It can be seen that after a short transition period, the pressure signal 


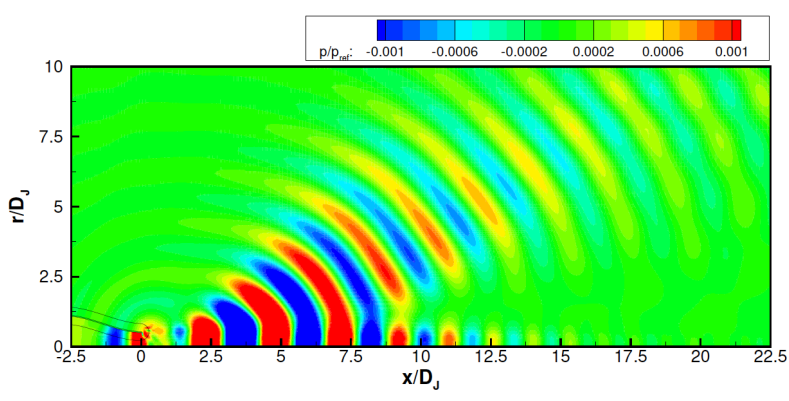

(a) Pressure, $\mathrm{St}=0.255$

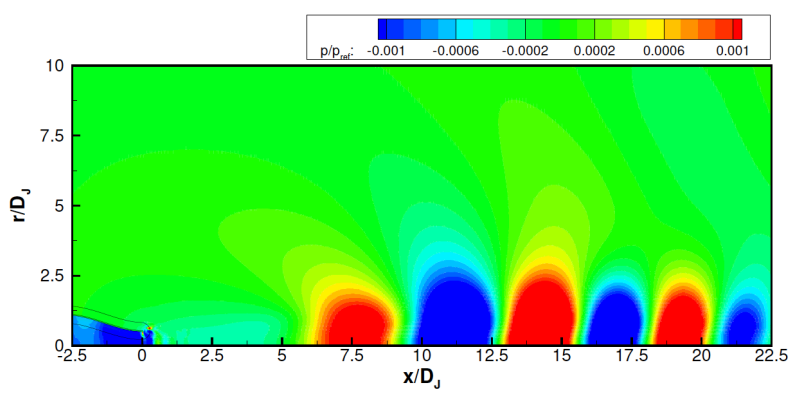

(c) Pressure, $\mathrm{St}=0.051$

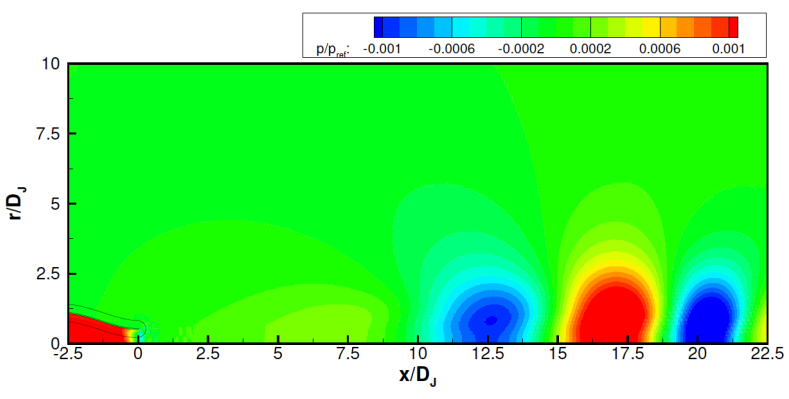

(e) Pressure, St $=0.0255$

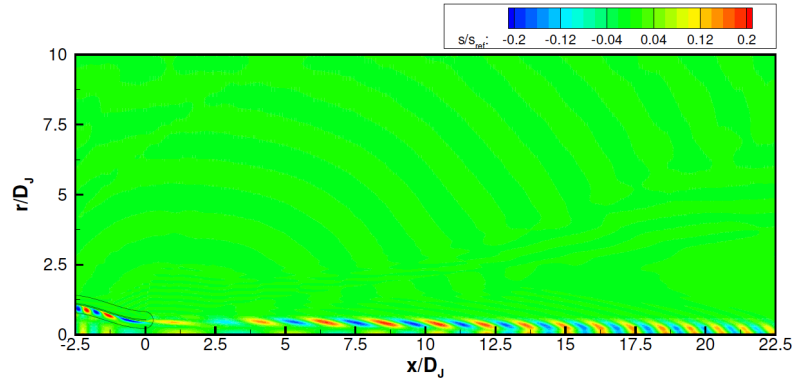

(b) Entropy, St $=0.255$

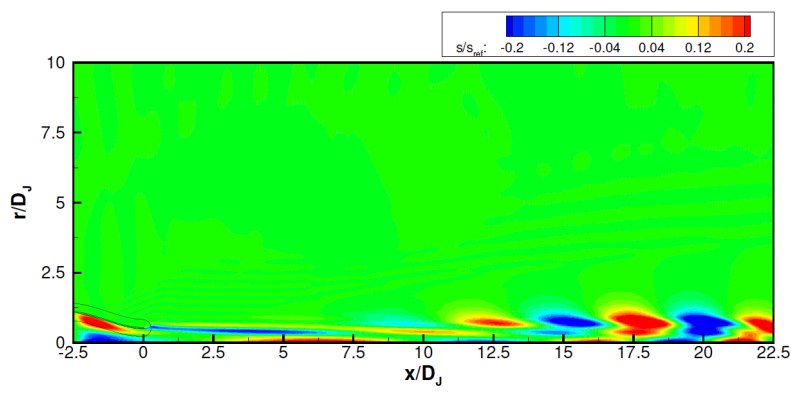

(d) Entropy, St $=0.051$

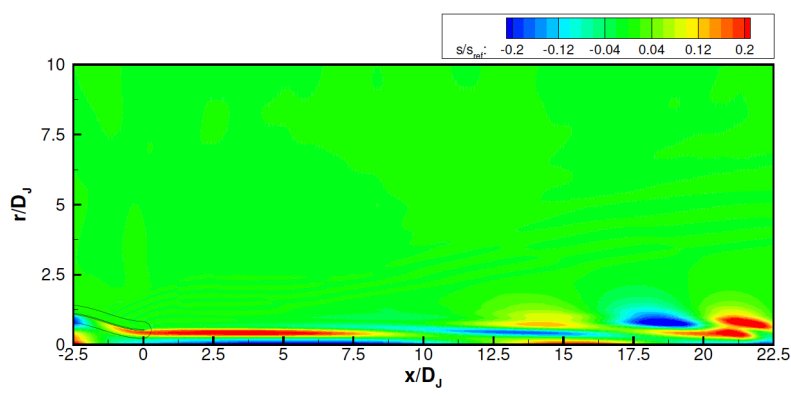

(f) Entropy, St $=0.0255$

Figure 12. Instantaneous flow-field results for pressure (left) and entropy (right) for three different Strouhal numbers: $\mathrm{St}=0.255$ (top), $\mathrm{St}=0.051$ (middle), and $\mathrm{St}=0.0255$ (bottom).

oscillates at the excitation frequency, which is a direct result of the linear model-formulation. It is also noted that the magnitude of the pressure signal is different for all three cases. We attribute this to the specification of the inflow perturbation, Eq. (6), whose rms-value is frequency-dependent.

\section{Summary and Conclusions}

Effects of nozzle-upstream entropy perturbations on the acoustic radiation from a heated jet are investigated. For this, a model problem is considered, in which a gas-turbine combustor discharges reaction products through a converging nozzle into the ambient environment. The turbulent reacting flow field in the combustor is computed using large-eddy simulation (LES), and the unsteady flow-field at the combustor exit is extracted to provide realistic inflow conditions to the jet-flow simulation. A steady-state RANS simulation is employed to compute the nozzle and the jet mean-flow. A linearized Euler formulation is used to simulate the spatio-temporal evolution of flow-field fluctuations for velocity, pressure, and entropy, and the coupling to the mean flow. The linearized Euler equations are solved in a generalized curvilinear coordinate system using a 4th-order accurate spatial discretization scheme.

Parametric studies are performed to investigate effects of frequency and amplitude of the nozzle-upstream 


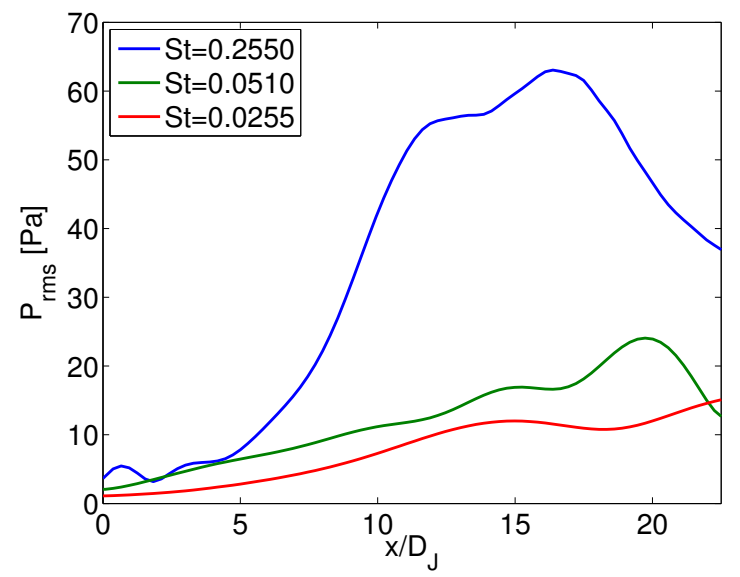

Figure 13. Near-field directivity at a distance $r / D_{J}=8.5$ along the axial direction.

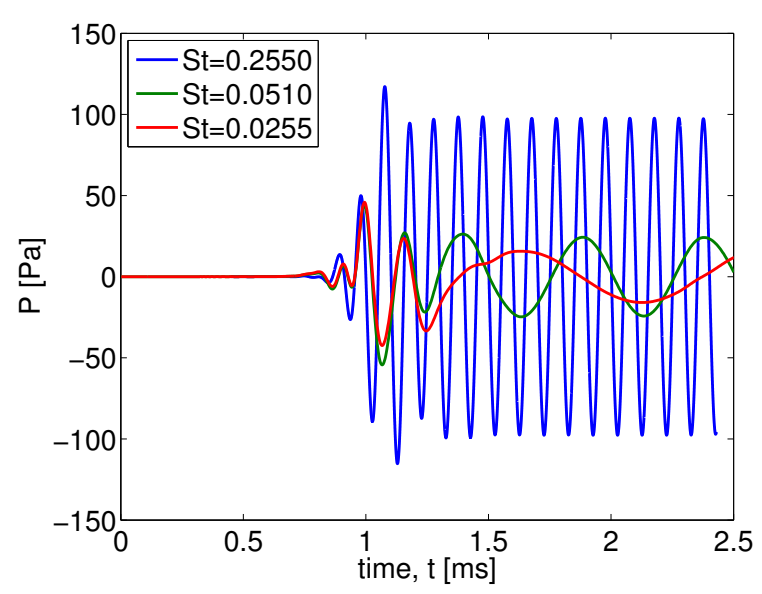

(a)

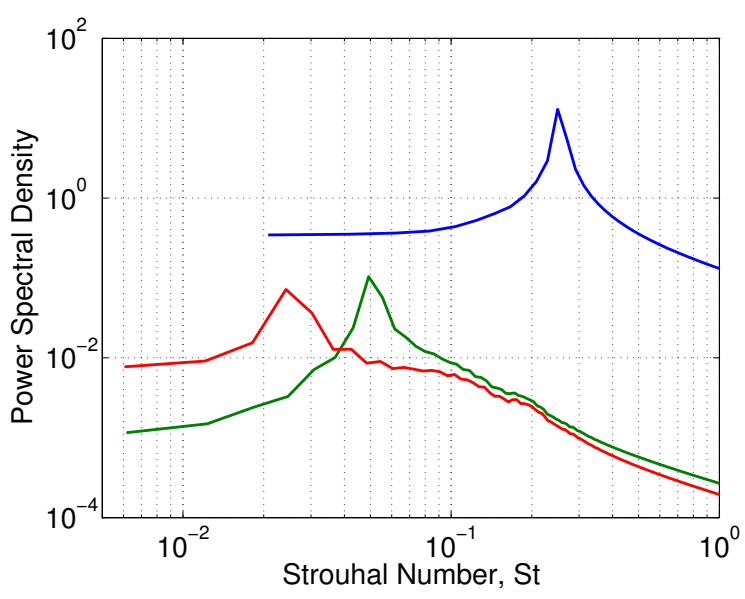

(b)

Figure 14. Comparison of pressure signal (a) and power spectral density (b) at measurement location $x / D_{J}=15$ and $r / D_{J}=8$.

entropy perturbations on the jet instability and the jet noise directivity. Simulation results show that the directivity is dependent on the perturbation frequency. Excitation around the preferred shear-layer instability leads to strong acoustic radiation in the $45^{\circ}$ forward direction, and the radiation angle decreases with decreasing excitation frequency.

Further research will focus on extending this analysis to different POD-excitation modes to investigate the sensitivity of the spatial structure on the jet instability. Another research direction addresses the consideration of the non-linear interaction between mean-flow, turbulence, and acoustic perturbations.

\section{Acknowledgments}

The authors gratefully acknowledge financial support through the Office of Naval Research under Grant No. N00014-10-1-0717 with Joseph Doychak as program manager. Helpful discussions with Brenda Henderson and Kailas Kailasanath regarding the model formulation and nozzle geometry are appreciated. 


\section{References}

${ }^{1}$ Tanna, H. K., Dean, P. D., and Burrin, R. H., "The generation and radiation of supersonic jet noise, Vol. III: Turbulent mixing noise data," AFAPL-TR-76-65-VOL III, AFAPL, 1976.

${ }^{2}$ Tam, C. K. W., "Supersonic jet noise," Ann. Rev. Fluid Mech., Vol. 27, 1995, pp. 17-43.

${ }^{3}$ Morris, P. J., Long, L. N., Scheidegger, T. E., and Boluriaan, S., "Simulation of supersonic jet noise," Int. J. Aeroacoustics, Vol. 1, No. 1, 2002, pp. 17-41.

${ }^{4}$ Brown, C. A., "Acoustics of excited jets - A historical perspective," NASA/TM-2005-213889, NASA, 2005.

${ }^{5} \mathrm{Lu}$, H. Y., "Effect of excitation on coaxial jet noise," AIAA J., Vol. 12, No. 2, 1983, pp. 214-220.

${ }^{6}$ Gerend, R. P., Kumasaka, H. A., and Roundhill, J. P., "Core engine noise," Progress in Astronautics and Aeronautics, Aeroacoustics: Jet amd Combustion Noise; Duct Acoustics, edited by H. T. Nagamatsu, 1975, pp. 305-326.

${ }^{7}$ Bechert, D. and Pfizenmaier, E., "On the amplification of broadband jet noise by a pure tone excitation," AIAA Paper 1976-489, 1976.

${ }^{8}$ Bechert, D. and Pfizenmaier, E., "Amplification of broad-band jet noise by a pure-tone excitation," J. Sound Vib., Vol. 43, No. 3, 1975, pp. 581-587.

${ }^{9}$ Berman, C. H., "Turbulence and noise characteristics of acoustically excited bypass jet flows," AIAA Paper 81-2009, 1981.

${ }^{10}$ Jubelin, B., "New experimental studies on jet noise amplifications," AIAA Paper 80-0961, 1980.

${ }^{11}$ Raman, G., Zaman, K. B. M. Q., and Rice, E. J., "Initial turbulence effect on jet evolution with and without tonal excitation," Phys. Fluids A, Vol. 1, No. 7, 1989, pp. 1240-1248.

${ }^{12}$ Ahuja, K. K. and Whiffen, M. C., "Tone excited jets, Part II: Flow visualization," J. Sound Vib., Vol. 102, 1985, pp. 63-69.

${ }^{13}$ Lepicovsky, J., Ahuja, K. K., and Burrin, R. H., "Tone excited jets, Part III: Flow measurements," J. Sound Vib., Vol. 102, No. 1, 1985, pp. 71-91.

${ }^{14}$ Ahuja, K. K. and Blakney, D. F., "Tone excited jets, Part IV: Acoustic measurements," J. Sound Vib., Vol. 102, No. 1, 1985, pp. 93-117.

${ }^{15}$ Zaman, K. B. M. Q., "Far-field noise of a subsonic jet under controlled excitation," J. Fluid Mech., Vol. 152, No. 1, 1985, pp. 83-111.

${ }^{16}$ Miles, J. H., "Separating turbofan engine noise sources using auto- and cross spectra from four microphones," AIAA J., Vol. 46, 2008, pp. 61-74.

${ }^{17}$ Miles, J. H., "Time delay analysis of turbofan engine direct and indireact combustion noise sources," J. Prop. Power, Vol. 25, No. 1, 2009, pp. 218-227.

${ }^{18}$ Poinsot, T. and Veynante, D., Theoretical and Numerical Combustion, R.T. Edwards, Inc., Philadelphia, PA, 2001.

${ }^{19}$ Knoop, P., Culick, F. E. C., and Zukoski, E. E., "Extension of the stability of motions in a combustion chamber by nonlinear active control based on hysteresis," Combust. Sci. Tech., Vol. 123, 1997, pp. 363-376.

${ }^{20}$ Burnley, V. S. and Culick, F. E. C., "Influence of random excitations on acoustic instabilities in combustion chambers," AIAA J., Vol. 38, No. 8, 2000, pp. 1403-1410.

${ }^{21}$ Lieuwen, T. C., "Experimental investigation of limit-cycle oscillations in an unstable gas turbine combustor," J. Prop. Power, Vol. 18, No. 1, 2002, pp. 61-67.

${ }^{22}$ Putnam, A. A., Combustion-Driven Oscillations in Industry, American Elsevier, New York, 1971.

${ }^{23}$ Marble, F. E. and Candel, S. M., "Acoustic disturbance from gas non-uniformities convected through a nozzle," J. Sound Vib., Vol. 55, No. 2, 1977, pp. 225-243.

${ }^{24}$ Meier, W., Weigand, P., and Duan, X. R., "Investigations of swirl flames in a gas turbine model combustor II. Turbulencechemistry interactions," Combust. Flame, Vol. 144, 2006, pp. 255-236.

${ }^{25}$ Pierce, C. D. and Moin, P., "Progress-variable approach for large-eddy simulation of non-premixed turbulent combustion," J. Fluid Mech., Vol. 504, 2004, pp. 73-97.

${ }^{26}$ Ihme, M., Cha, C. M., and Pitsch, H., "Prediction of local extinction and re-ignition effects in non-premixed turbulent combustion using a flamelet/progress variable approach," Proc. Combust. Inst., Vol. 30, 2005, pp. 793-800.

${ }^{27}$ Bowman, C. T., Hanson, R. K., Davidson, D. F., Gardiner, W. C., Lissianski, V., Smith, G. P., Golden, D. M., Frenklach, M., and Goldenberg, M., "GRI-Mech 2.11," 1997, available from http://www.me.berkeley.edu/gri-mech/.

${ }^{28}$ Plumblee, H. E., Burrin, R. H., Lau, J. C., Morfey, C. L., Morris, P. J., Smith, D. M., Tanna, H. K., Tester, B. J., and Whiffen, M. C., "The generation and radiation of supersonic jet noise, Vol. II: Studies of jet noise, turbulence structure and laser velocimetry," AFAPL-TR-76-65-VOL II, AFAPL, 1976.

${ }^{29}$ Monkewitz, P. A. and Sohn, K. D., "Absolute instability in hot jets," AIAA J., Vol. 26, No. 8, 1988, pp. 911-916.

${ }^{30} \mathrm{Tam}, \mathrm{C} . \mathrm{K} . \mathrm{W}$. and Webb, J. C., "Dispersion-relation-preserving finite difference schemes for computational acoustics," J. Comp. Phys., Vol. 107, No. 2, 1993, pp. 262-281.

${ }^{31} \mathrm{Hu}$, F. Q., Hussaini, M. Y., and Manthey, J. L., "Low-dissipation and low-dispersion Runge-Kutta schemes for computational acoustics," J. Comp. Phys., Vol. 124, No. 1, 1996, pp. 177-191.

${ }^{32}$ Tam, C. K. W. and Dong, Z., "Wall boundary conditions for high-order finite-difference schemes in computational aeroacoustics," Theoretical and Computational Fluid Dynamics, Vol. 6, No. 5, 1994, pp. 303-322.

${ }^{33}$ Barone, M. F. and Lele, S. K., "Receptivity of the compressible mixing layer," J. Fluid Mech., Vol. 540, 2005, pp. 301-335.

${ }^{34}$ Brown, D. L., Henshaw, W. D., and Quinlan, D. J., "Overture: Object-oriented tools for overset grid applications," AIAA-99-3130, 1999.

${ }^{35}$ Visbal, M. R. and Gaitonde, D. V., "On the use of higher-order finite-difference schemes on curvilinear and deforming meshes," J. Comp. Phys., Vol. 181, No. 1, 2002, pp. 155-185.

${ }^{36}$ Agarwal, A., Mani, R., and Morris, P., "Calculation of sound propagation in nonuniform flows: Suppression of instability waves," AIAA J., Vol. 42, No. 1, 2004, pp. 80-88. 